\title{
Retalho bilobado na reconstrução das clinodactilias da mão: Descrição da técnica e avaliação do resultado
}

\section{Bilobed Flap in Hand Clinodactyly Reconstruction: Technique Description and Result Appraisal}

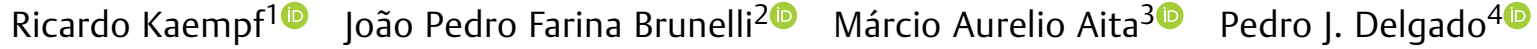 \\ Francisco Soldado ${ }^{5 \odot}$ Enrique Vergara6®
}

${ }^{1}$ Departamento de Cirurgia da Mão, Hospital da Santa Casa de

Misericórdia, Porto Alegre, RS, Brasil

2 Departamento de Cirurgia da Mão da Santa Casa de Misericórdia,

São Paulo, SP, Brasil

${ }^{3}$ Departamento de Cirurgia da Mão, Faculdade de Medicina do ABC,

Santo André, SP, Brasil

${ }^{4}$ Departamento de Cirurgia da Mão, Hospital Universitário Madrid Montepríncipe, Universidad San Pablo CEU, Boadilla del Monte, Madrid, Espanha

\author{
Endereço para correspondência Ricardo Kaempf, MD, Departamento \\ de Cirurgia da Mão, Santa Casa de Misericórdia Hospital, Rua Leopoldo \\ Bier, 825 / 301, 90620-100, Porto Alegre, RS, Brasil \\ (e-mail: ricardokaempf@gmail.com). \\ ${ }^{5}$ Departamento de Cirurgia da Mão, Universitat de Barcelona, \\ Barcelona, Espanha \\ ${ }^{6}$ Departamento de Ortopedia e Traumatologia, Especialidade Mão, \\ Universidad Nacional de Colombia, Colômbia
}

Rev Bras Ortop 2022;57(4):642-648.

\section{Resumo \\ Palavras-chave \\ - clinodactilia \\ - dedos/anormalidades \\ - retalhos cirúrgicos \\ - osteotomia}

Objetivo A clinodactilia é uma deformidade congênita da mão, caracterizada por desvio angular coronal, podendo ocorrer em polegares ou dedos. O tratamento cirúrgico é indicado para angulações severas. Dentre as técnicas descritas, uma das opções consiste no alinhamento ósseo, através de osteotomia em cunha de adição da falange anômala. Essa manobra de alinhamento gera uma dificuldade de cobertura cutânea, juntamente com a tensão das partes moles no lado côncavo da deformidade. Sendo assim, algum tipo de retalho cutâneo é necessário para o fechamento adequado da ferida cirúrgica. Objetivamos demonstrar a técnica citada e avaliar os resultados do retalho bilobado no tratamento das clinodactilias da mão.

Métodos Estudo retrospectivo entre janeiro de 2008 e janeiro de 2015. Foram incluídos cinco pacientes no estudo, nove dedos foram submetidos à cirurgia. Tiveram indicação cirúrgica os pacientes com desvios angulares nos dedos acima de 30 graus. Não foram excluídos do estudo os pacientes com deformidades no polegar, nem aqueles com deformidades associadas a síndromes. Foram avaliados os resultados funcionais e estéticos com o uso desta técnica, assim como as complicações e o grau de satisfação dos familiares. Resultados Todos os pacientes apresentaram resultados funcionais e estéticos satisfatórios, com cicatrização cutânea média de 18,6 dias. Entre os nove dedos

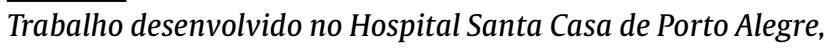

Porto Alegre (RS) Brasil.

recebido

30 de Novembro de 2020

aceito

11 de Fevereiro de 2021

published online

Outubro 1, 2021
DOI https://doi.org/

10.1055/s-0041-1731797.

ISSN 0102-3616.
(C) 2021. Sociedade Brasileira de Ortopedia e Traumatologia. All rights reserved.

This is an open access article published by Thieme under the terms of the Creative Commons Attribution-NonDerivative-NonCommercial-License, permitting copying and reproduction so long as the original work is given appropriate credit. Contents may not be used for commercial purposes, or adapted, remixed, transformed or built upon. (https://creativecommons.org/ licenses/by-nc-nd/4.0/)

Thieme Revinter Publicações Ltda., Rua do Matoso 170, Rio de Janeiro, RJ, CEP 20270-135, Brazil 
submetidos à cirurgia, apenas um dos pacientes apresentou comprometimento vascular na porção distal do primeiro lobo do retalho, porém sem necrose ou necessidade de qualquer procedimento adicional. Os pacientes foram acompanhados durante um intervalo mínimo de 12 meses. Nenhuma deformidade ocorreu durante o período de observação.

Conclusão O uso do retalho bilobado no tratamento das clinodactilias da mão é uma boa opção para cobertura cutânea após a realização de osteotomia.

\begin{abstract}
Keywords

- clinodactyly

- fingers/abnormalities

- surgical flaps

- osteotomy

Objective Clinodactyly is a congenital hand deformity that is characterized by coronal angular deviation and may occur in thumbs or fingers. Surgical treatment is indicated for severe angulations. Among the described techniques, one of the options consists of bone alignment by wedge-shaped addition osteotomy of the anomalous phalanx. Such alignment maneuver creates a problem in skin cover, along with soft-tissue tension at the concave aspect of the deformity. Hence, some sort of skin flap is required for the adequate operative wound closure. We aim to demonstrate the aforementioned technique and to assess the results of bilobed flap in the treatment of hand clinodactyly.

Methods Retrospective study conducted between January 2008 and January 2015. Five patients were included in the study, including nine operated digits. Surgical indication consisted of angular deviations $>30^{\circ}$. Neither patients with thumb deformities nor those with deformities associated to syndromes were excluded from the study. We assessed the functional and cosmetic outcomes of the technique, as well as complications and the satisfaction rates of the family.

Results All patients had satisfactory functional and cosmetic results, with a mean skin healing of 18.6 days. Among nine operated digits, only one of the patients presented vascular compromise at the distal portion of the first flap lobe, albeit without necrosis or the need for any additional procedure. Patients were followed up on a minimum of 12-month interval. No deformity recurred during the observation period.

Conclusion Bilobed flap for the treatment of hand clinodactyly is a good option for skin cover after the osteotomy.
\end{abstract}

\section{Introdução}

Clinodactilia é uma deformidade congênita da mão, caracterizada por desvio angular coronal, podendo ocorrer em polegares ou dedos. A deformidade do dedo mínimo é frequente e, muitas vezes, leve, se manifestando como uma deformidade isolada. As angulações $>20^{\circ}$ geram alterações funcionais com sobreposição dos dedos durante a flexão. ${ }^{1-4}$ As deformidades do polegar geralmente são mais graves, podendo estar associadas às síndromes de: Rubinstein-Taybi, Pfeiffer, Apert, e Patau, entre outras. ${ }^{1-4}$ Na maioria dos casos, ocorre um desvio radial; estas deformidades também são conhecidas como "polegar do carona." 5

As angulações graves geralmente mostram a substituição de uma falange normal por outra com formato anormal, podendo esta apresentar forma triangular ou trapezoidal. $O$ tratamento cirúrgico é indicado para esses pacientes. Uma das opções consiste no alinhamento ósseo, por meio de osteotomia em cunha adicional da falange anômala e a fixação com fios de Kirschner. ${ }^{6}$ Essa manobra de alinhamento gera um problema na cobertura cutânea e tensão das partes moles no lado côncavo da deformidade. Ainda assim, algum tipo de retalho cutâneo é necessário para o fechamento adequado da ferida cirúrgica. ${ }^{7}$

O uso do retalho bilobado, conforme descrito por Esser, ${ }^{8} \mathrm{e}$ outras alterações, ${ }^{9}$ permite a redistribuição cutânea, levando a pele redundante do lado convexo para o lado côncavo da deformidade. Essa técnica permite o alinhamento dos dedos, sem a necessidade de enxerto de pele ou retalhos de sítios distantes.

O objetivo deste estudo é detalhar a técnica do retalho bilobado no tratamento cirúrgico da clinodactilia, conforme descrito por Cerqueiro-Mosquera e Fleming ${ }^{5}$ no ano de 2000, avaliando os resultados de uma série de nove dedos em cinco pacientes.

\section{Material e Métodos}

O projeto foi submetido ao Comitê de Ética em Pesquisa (CEP) da instituição, sob o número CAAE 87964217.4.0000.5335.

Avaliamos retrospectivamente todos os pacientes tratados cirurgicamente em nosso serviço para clinodactilia com 
retalho bilobado, durante o período de janeiro de 2008 até janeiro de 2015. Foram incluídos neste estudo cinco pacientes, com nove dedos tratados cirurgicamente.

A técnica cirúrgica foi indicada apenas para desvios angulares dos dedos $>30^{\circ}$. Não foram excluídas do estudo as deformidades no polegar nem aquelas associadas às síndromes. As anomalias ortopédicas associadas a outros dedos, como a sindactilia, também não foram excluídas.

O procedimento foi realizado sob anestesia geral, com aplicação de torniquete pneumático no membro superior. Quanto à técnica cirúrgica empregada, ela começa pelo desenho da incisão, que deve ser realizado rotineiramente, a fim de se obter um fechamento primário mais seguro. No local planejado para incisão, é traçada uma linha transversal no ponto da deformidade máxima, na face côncava do dedo e alinhada com a prega de extensão interfalangeana. Essa incisão inicia-se na junção entre a pele dorsal e a pele glabra (sem pelos), seguindo em direção ao lobo dorsal do retalho. A largura do lobo dorsal deve preencher o tamanho do espaço que será criado no lado côncavo no alinhamento dos dedos. O lobo dorsal se estende distalmente da prega de extensão interfalangeana até $2 / 3$ da distância da prega ungueal. O lobo secundário deve ser executado em direção à palma da mão, no lado convexo da deformidade, devendo ter altura semelhante à do mesmo lobo, porém com largura menor (-Fig. 1).

Deve-se ter cuidado durante a dissecção dorsal, a fim de manter intacto o tecido tenossinovial do aparelho extensor, juntamente com as principais veias dorsais. Os lobos do retalho incluem a pele e o tecido subcutâneo, devendo-se ter o cuidado no lado convexo do lobo secundário em manter o feixe neurovascular íntegro em sua posição.

A incisão que é criada para o retalho bilobado, deve ser larga o suficiente para que o delta anômalo ou a falange

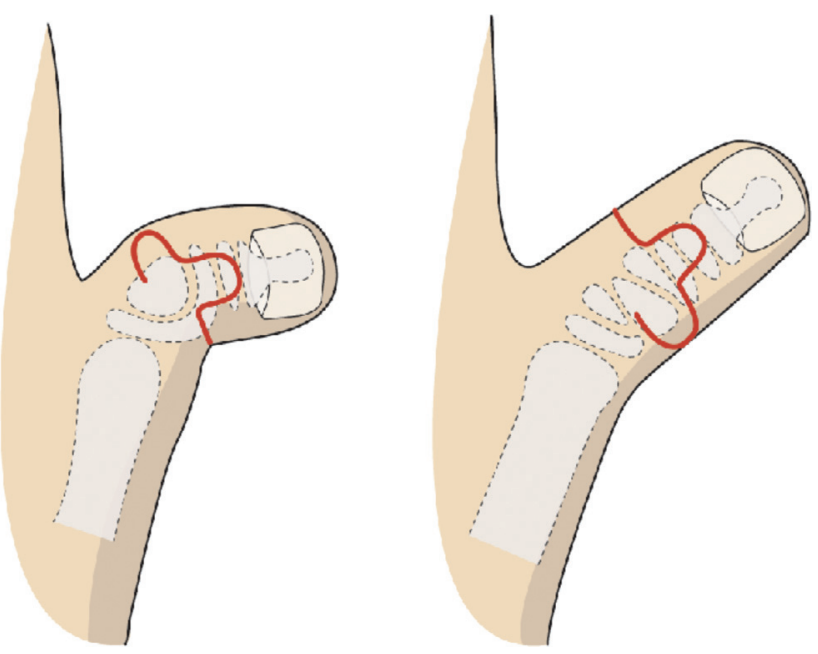

Fig. 1 Esquema do tratamento cirúrgico da clinodactilia com retalho bilobado. Começa com uma linha transversal na borda côncava do dedo, que é o ponto de deformidade máxima. O lobo dorsal deve ser do tamanho do espaço criado na borda côncava com o dedo alinhado. O lobo secundário deve ser executado em direção à palma da mão no lado convexo da deformidade, devendo ter uma altura semelhante à do primeiro lobo, embora menos largo. trapezoidal seja visualizado de ambos os lados do tendão extensor, facilitando a osteotomia. A osteotomia é realizada transversalmente na falange, a fim de que a correção da deformidade crie uma cunha de abertura na face côncava da deformidade. Após a osteotomia, a falange é fixada com um ou dois fios de Kirschner. Não foi empregado o enxerto ósseo em nossa série de casos.

Os lobos do retalho sofrem uma rotação de $90^{\circ}$ para o fechamento, de modo que o lobo dorsal feche o defeito no lado côncavo e o lobo convexo secundário cubra o defeito dorsal. O lado convexo é basicamente fechado, sem tensão ou necessidade de enxerto de pele. Antes do fechamento dos retalhos, o torniquete pneumático é desinflado para a realização da hemostasia. $O$ paciente é inicialmente imobilizado com gesso de braço longo, incluindo o dedo operado, que, após a primeira semana, é substituído por uma tala de alumínio para dedo. Os fios de Kirschner são removidos após 8 semanas. Depois disso, é prescrito um programa doméstico de exercícios de mobilização, que geralmente são suficientes para a recuperação da mobilidade. Os resultados cosméticos e funcionais são excelentes com esta técnica, não dando origem a complicações.

\section{Resultados}

Entre os cinco pacientes incluídos em nossa série, um apresentava síndrome de Apert; outro a síndrome de Pfeiffer (-Fig.2), dois apresentavam síndrome de Rubinstein-Taybi ambos eram gêmeos homozigotos e um era portador de clinodactilia isolada (-Figs. 3 e 4). Todos os pacientes eram do sexo masculino, com idade média de 4 anos e 1 mês (variando de 2 anos e 5 meses a 7 anos e 4 meses), no momento em que foram submetidos à cirurgia. No total foram operados nove dedos, incluindo oito polegares e um dedo indicador. Todos os pacientes apresentaram desvio radial e o ângulo médio foi de $67,2^{\circ}$ (variando de $60-80^{\circ}$ ). Os pacientes foram acompanhados clinicamente, com uma média de 18,6 meses (variação de 12-24 meses). Por se tratar de uma amostra de pacientes pediátricos, nenhuma dominância foi determinada no momento da cirurgia (-Tabela $\mathbf{1}$ ).

Não houve complicações perioperatórias graves ou pósoperatórias imediatas em relação à técnica. O procedimento expressou o fechamento primário de todos os dedos operados. Todos os 9 dedos evoluíram com cicatrização total do retalho em 18,6 dias (variando de 14-28 dias). Todos os retalhos resistiram e apenas um paciente apresentou comprometimento distal de parte do primeiro lobo. Nesse caso, formou-se uma crosta, seguida de cicatrização de segunda intenção.

Três pacientes com deformidade bilateral foram submetidos a cirurgia em ambas as mãos no mesmo dia. Apenas um paciente com deformidade bilateral do polegar foi operado em duas etapas, em virtude da sindactilia do terceiro e quarto dedos, sendo abordada conjuntamente com a clinodactilia do polegar. Nenhum paciente precisou de um segundo procedimento. Todos os pacientes apresentaram cosmese boa ou excelente, sendo que não foi observada nenhuma recidiva de deformidade no acompanhamento mínimo de 12 meses. Na 


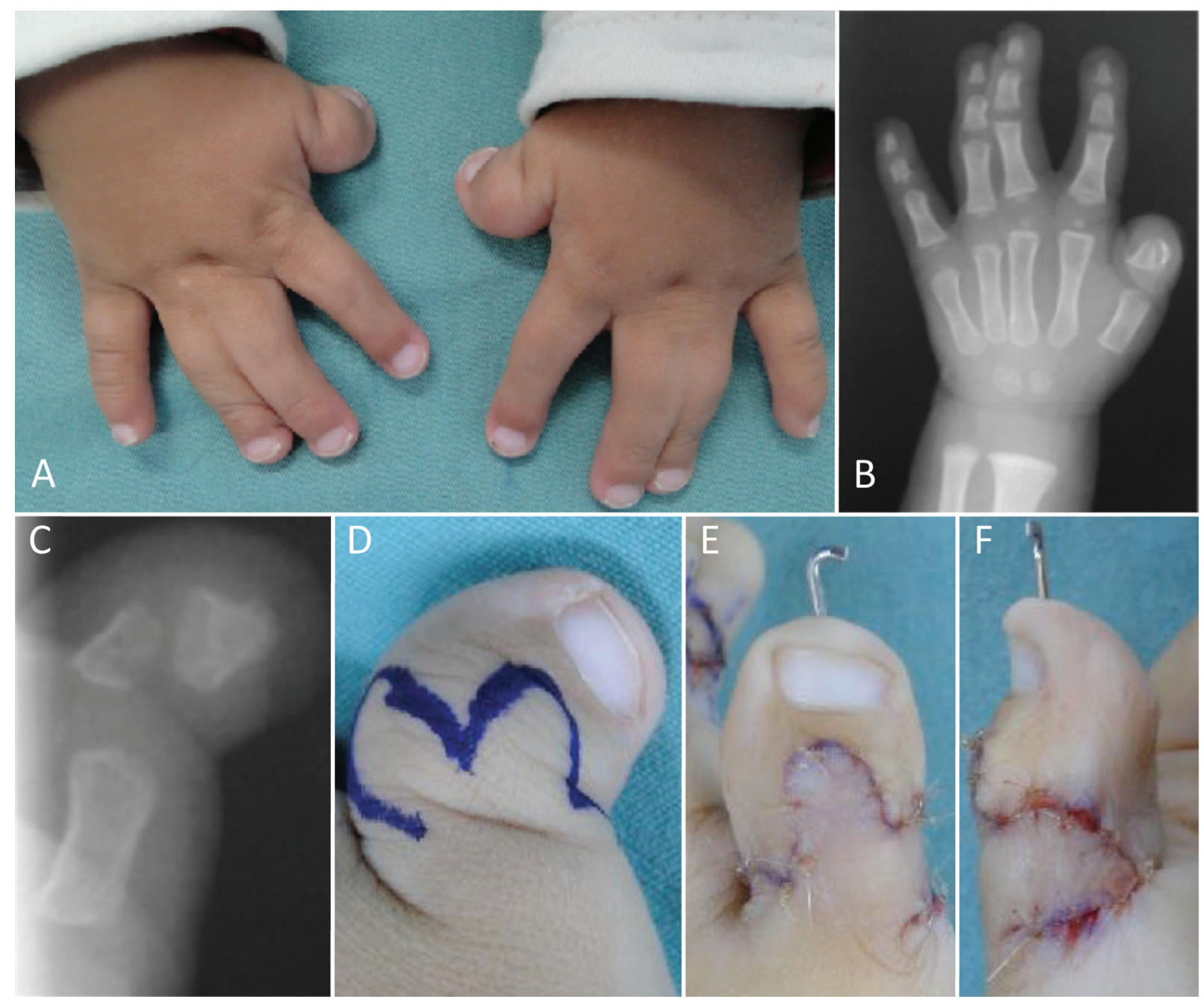

Fig. 2 Paciente com síndrome de Pfeiffer apresentando alterações bilaterais nas mãos, com deformidade típica do polegar do carona, associada à sindactilia do terceiro e quarto dedos (A). Radiografia mostrando falange proximal em delta do polegar e hipoplasia das falanges médias dos dedos longos (B). A radiografia do polegar detalha clinodactilia e a falange em delta (C). Planejamento cirúrgico com retalho cutâneo bilobado (D). Elevação e rotação de retalho bilobado com cobertura cutânea completa (E e F).

avaliação subjetiva, todos os pacientes e familiares relataram estar satisfeitos com o resultado cirúrgico.

\section{Discussão}

A clinodactilia é uma anomalia congênita caracterizada por um desvio lateral excessivo do dedo. Em dedos longos, isso geralmente ocorre devido ao desenvolvimento anormal da falange média, levando à deformidade da articulação interfalangeana distal. Excepcionalmente, pode levar ao desvio da articulação interfalangeana proximal, em virtude de uma anormalidade da falange proximal. ${ }^{1-4} \mathrm{~A}$ clinodactilia é causada principalmente por uma falange em delta, que é definida por sua forma triangular, com uma epífise longitudinal em vez de transversal, estando localizada no lado mais curto da falange. A epífise anormal altera o crescimento ósseo, causando um efeito semelhante ao de um grampo. As falanges em delta exibem um formato em $\mathrm{D}$ ou circular nas radiografias. Um desvio progressivo lateral do dedo ocorre com o crescimento. ${ }^{2,6}$

O dedo mais frequentemente afetado é o quinto, apresentando deformidade radial, em que é mais comum o comprometimento bilateral. Esses casos geralmente apresentam uma história familiar positiva, devido a uma expressividade variável e a uma característica autossômica dominante. $O$ segundo dedo mais frequentemente afetado é o dedo indicador, mostrando desvio ulnar ou radial. Os procedimentos cirúrgicos são indicados para angulações severas e alterações funcionais, que provoquem uma sobreposição do dedo durante a flexão, o que geralmente ocorre com angulações $>30^{\circ}$. 2,3,10

O tratamento para polegar do carona é o mesmo usado para falange em delta. ${ }^{11}$ Poucos estudos avaliaram o resultado do tratamento cirúrgico da clinodactilia do polegar, sendo que a maioria relatou a recorrência da deformidade em mais de $30 \%$ dos pacientes. ${ }^{11}$ 

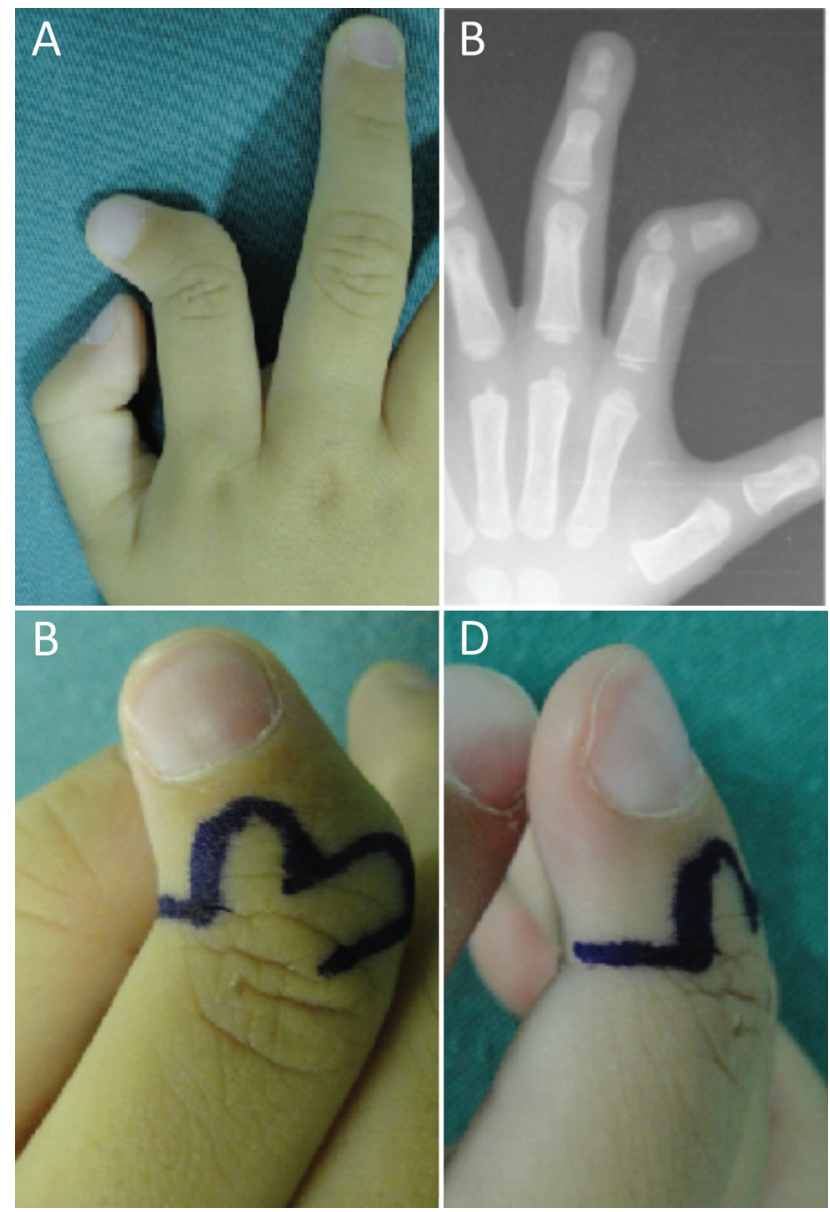

Fig. 3 Paciente com clinodactilia do dedo indicador. Deformidade isolada não associada a nenhuma síndrome (A). A radiografia prova o desenvolvimento anormal da falange média. Falange em delta (B). Planejamento cirúrgico com retalho cutâneo bilobado (C e D).

Assim sendo, nossa série incluiu pacientes com a síndrome de Apert, na qual o polegar é sempre curto e desviado radialmente, síndrome de Pfeiffer, na qual as mãos são afetadas em quase $80 \%$ dos casos e as alterações são bastante variáveis. Apenas $20 \%$ dos pacientes com essa síndrome apresentam a deformidade típica do polegar do carona. A outra síndrome encontrada na nossa série é a síndrome de Rubinstein-Taybi, uma rara patologia dismórfica descrita em 1963. ${ }^{12,13}$ A mão aparece com o polegar alargado, com desvio radial, devido à presença de uma falange em delta proximal, formando o polegar do carona.

A clinodactilia foi classificada por Burke e Flatt. ${ }^{14}$ Ali et al. ${ }^{4}$ a classificaram de acordo com a gravidade da deformidade.

Seja qual for a causa, a cirurgia corretiva para clinodactilia visa melhorar a função e a cosmese. Diferentes procedimentos cirúrgicos foram descritos, sendo que a escolha da técnica depende da patologia subjacente, do grau de deformidade, da contratura dos tecidos moles, da idade e da preferência do cirurgião. 0 procedimento deve abordar o alinhamento ósseo, o alongamento dos tecidos moles e a cobertura cutânea. ${ }^{1,7}$
Diferentes procedimentos ósseos foram propostos. Burke e Flatt $^{14}$ descreveram a osteotomia de ressecção, ou em cunha de fechamento, um procedimento mais simples que traz uma armadilha, provocando um encurtamento em um dedo já encurtado. Carstam e Theander ${ }^{15}$ preconizam uma osteotomia em cunha de inversão, na qual uma cunha é removida de um lado da falange e colocada do outro lado. Vickers ${ }^{7}$ propõe a abertura fisária através da incisão mediolateral no ápice da deformidade e a interposição de gordura para o tratamento precoce da clinodactilia. A deformidade, portanto, não é corrigida durante a cirurgia, mas durante o crescimento ósseo longitudinal. Esse tratamento não é indicado para os pacientes com mais de 10 anos de idade ou com deformidades no polegar. Jain et al. ${ }^{16}$ empregaram uma osteotomia em forma de cúpula, em 8 dos 13 pacientes operados, relatando recorrência da deformidade angular em 5 pacientes. Jones ${ }^{17}$ descreveu uma osteotomia em cunha de adição ou abertura, com a vantagem de criar um dedo maior, juntamente com o alongamento ósseo e das partes moles, com cobertura cutânea por meio da zetaplastia ou enxerto ósseo de espessura total. Al Qattan ${ }^{18}$ aponta o uso do procedimento de Vickers para o tratamento precoce, a osteotomia em cunha de adição para os pacientes idosos e com deformidades mais graves. Outras técnicas descritas, como a fixação simples com fios de Kirschner, artrodese primária e a capsulotomia isolada, foram abandonadas devido aos resultados desfavoráveis.

O alinhamento de uma deformidade angular grave do dedo, requer cuidados concomitantes com a cobertura cutânea, especialmente nos casos de osteotomia em cunha de adição. A necessidade de liberação das partes moles no lado côncavo da deformidade pode surgir durante o procedimento. Os ligamentos colaterais e a cápsula articular podem ser liberados ou alongados, permitindo o correto posicionamento dos dedos. ${ }^{6}$ Vários retalhos diferentes foram descritos, o objetivo é a cicatrização rápida com pele de boa qualidade, cicatrizes mínimas e ausência da necessidade de enxerto de pele de uma região distante ou da rotação de retalhos cutâneos. ${ }^{4}$ A zetaplastia pode produzir uma cobertura cutânea satisfatória para os casos de osteotomia em cunha de ressecção. ${ }^{13}$

O retalho bilobado foi originalmente descrito por Esser em $1918,^{8}$ e devido à sua versatilidade, tem sido empregado na reconstrução dos defeitos em diferentes regiões anatômicas. Tem sido relatada a sua utilização na correção da deformidade da mão com inclinação radial, ${ }^{12}$ bem como na cobertura cutânea de cisto mucoso localizado na articulação interfalangeana distal na pós-correção. ${ }^{19,20} \mathrm{O}$ ângulo entre os lobos do retalho foi originalmente descrito como sendo de $90^{\circ}$; entretanto, diversos autores criaram variações de uso e forma, permitindo assim a possibilidade de variação do ângulo entre 45 a $180^{\circ}$, dependendo da aplicação. ${ }^{20,21} \mathrm{~A}$ nossa série seguiu a técnica proposta por Cerqueiro-Mosqueira e Fleming, ${ }^{5}$ utilizando o retalho bilobado para auxiliar na reconstrução da clinodactilia. Esse retalho é obtido de uma forma simples e cuidadosa, aproveitando a redundância cutânea de uma região, agregando cobertura cutânea de 


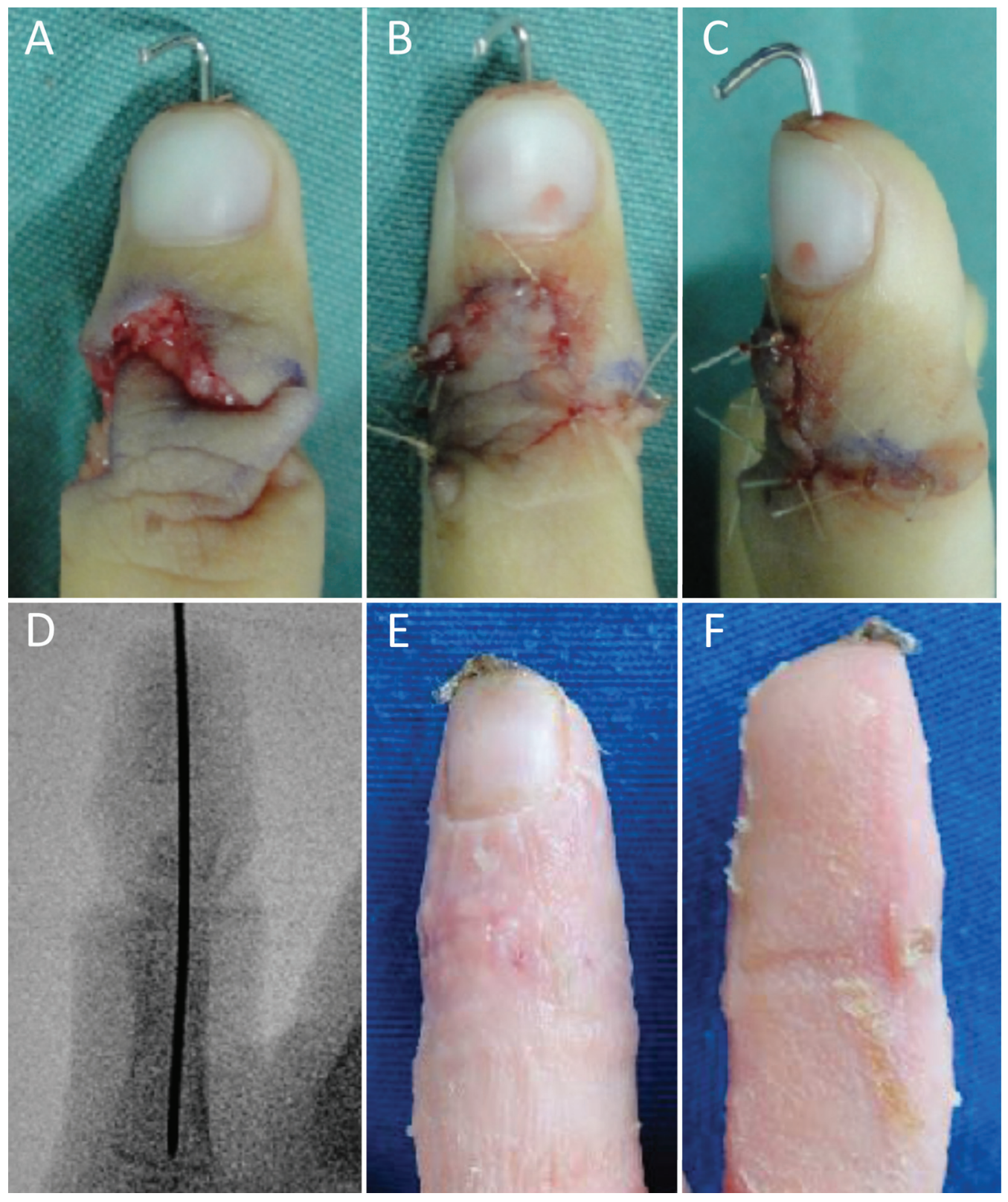

Fig. 4 Mesmo paciente da figura anterior. Elevação e rotação do retalho bilobado, com cobertura cutânea completa (A, B e C). Osteotomia da falange em delta e a fixação com fios de Kirschner (D). Cicatrização da pele após duas semanas de pós-operatório (E e F).

qualidade para outra região. O desenho geométrico é descomplicado e reproduzível, reduzindo a curva de aprendizado. 0 resultado cosmético da cicatriz foi excelente, já que o retalho cutâneo permite o fechamento da pele sem tensão.

O retalho bilobado apresenta vantagens claras sobre outras técnicas de redistribuição cutânea em defeitos angulares, devido à simplicidade da criação e ao uso potencial de pele redundante local. É um procedimento seguro, permitindo uma cobertura cutânea adequada durante a cirurgia, sem tensão sobre a sutura, evitando assim a necessidade de enxerto cutâneo ou a cicatrização de segunda intenção. Sua forma geométrica simples transmite confiabilidade, permitindo um procedimento rápido, único e definitivo. O resultado cosmético final foi excelente, proporcionando cicatrização em curto espaço de tempo. ${ }^{5,20}$ É difícil encontrar um cirurgião com pelo menos razoável experiência no tratamento da clinodactilia de mãos, por sua raridade. Realizamos o retalho bilobado em pacientes com diferentes síndromes com bons resultados, principalmente em doenças que apresentam tendência ao desenvolvimento de cicatriz queloide. ${ }^{13}$ Os pacientes apresentaram evolução cosmética boa ou excelente. Não houve recidiva de deformidade, durante um acompanhamento mínimo de 12 meses. Deve ser levado em conta, que esse tempo de acompanhamento é muito curto para dizer se vai haver alguma deformidade residual; entretanto, esse tipo de avaliação foi a opção escolhida, pois a ênfase deste estudo está na técnica de retalho cutâneo. 


\begin{tabular}{|c|c|c|c|c|c|c|c|c|c|}
\hline & $z$ & $z$ & 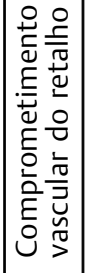 & $z$ & $z$ & $z$ & $z$ & $z$ & $z$ \\
\hline 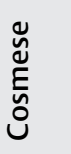 & $\begin{array}{l}0 \\
0 \\
\infty\end{array}$ & 芯 & 芯 & 舟 & 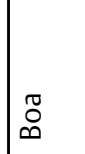 & 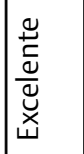 & 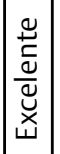 & 串 & 串 \\
\hline 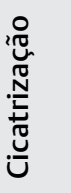 & \begin{tabular}{|l}
$E$ \\
$\bar{\omega}$ \\
$m$ \\
$m$
\end{tabular} & \begin{tabular}{|l}
$E$ \\
$\tilde{U}$ \\
$m$
\end{tabular} & \begin{tabular}{|l}
$E$ \\
$\tilde{\omega}$ \\
$\sim$
\end{tabular} & $\begin{array}{l}E \\
\tilde{U} \\
\sim \\
\sigma\end{array}$ & $\begin{array}{l}E \\
\stackrel{E}{U} \\
\sim\end{array}$ & $\begin{array}{l}E \\
\tilde{U} \\
\sim \\
\sim\end{array}$ & $\mid \begin{array}{c}E \\
\bar{U} \\
\sim\end{array}$ & $\begin{array}{l}E \\
\dot{U} \\
\tilde{m}\end{array}$ & $\begin{array}{l}E \\
\stackrel{E}{ } \\
m\end{array}$ \\
\hline 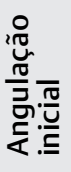 & 8 & 우 & $\infty$ & $\stackrel{\text { L }}{\wedge}$ & 늣 & ڤn & 인 & 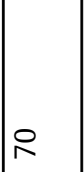 & 8 \\
\hline 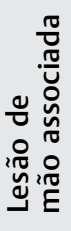 & 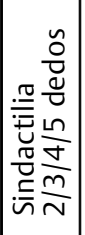 & 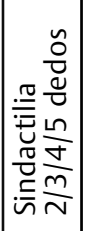 & 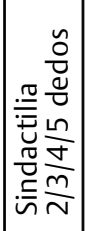 & 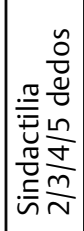 & $z$ & $z$ & $z$ & $z$ & $z$ \\
\hline$\stackrel{\circ}{\stackrel{*}{*}}$ & 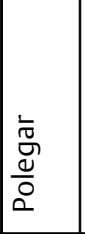 & 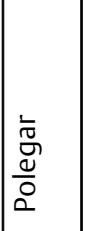 & $\begin{array}{l}\frac{\bar{\sigma}}{0} \\
\frac{\mathbb{U}}{0} \\
\therefore\end{array}$ & 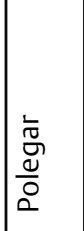 & 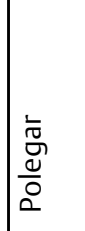 & \begin{tabular}{|l}
$\frac{\overline{0}}{0}$ \\
$\frac{\mathbb{U}}{0}$ \\
0
\end{tabular} & 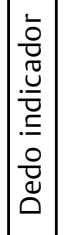 & $\begin{array}{l}\frac{\bar{\pi}}{0} \\
\frac{\mathbb{d}}{0} \\
\end{array}$ & $\begin{array}{l}\frac{1}{\mathrm{~T}} \\
\frac{\mathrm{U}}{0} \\
\end{array}$ \\
\hline 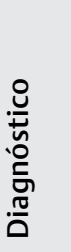 & 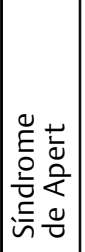 & 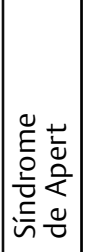 & 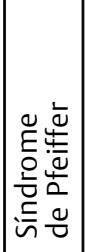 & 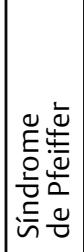 & 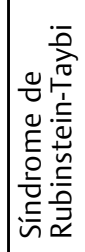 & 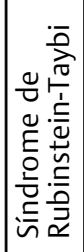 & 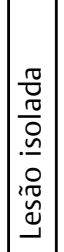 & 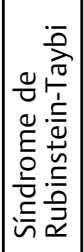 & 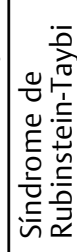 \\
\hline$\stackrel{\circ}{\stackrel{0}{0}}$ & 0 & س & 0 & س & o & س & ш & D & س \\
\hline $\begin{array}{l}\text { 원 } \\
\text { 㥕 }\end{array}$ & $\Sigma$ & $\Sigma$ & $\Sigma$ & $\Sigma$ & $\Sigma$ & $\Sigma$ & $\Sigma$ & $\Sigma$ & $\Sigma$ \\
\hline $\begin{array}{l}\frac{\tilde{D}}{\tilde{\pi}} \\
\frac{\pi}{0}\end{array}$ & 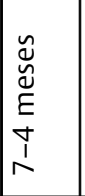 & 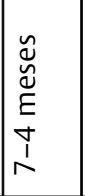 & 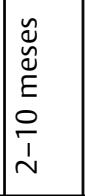 & 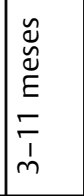 & 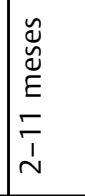 & $\begin{array}{l}\breve{y} \\
\dddot{u} \\
\underline{E} \\
\check{\check{\nu}} \\
\sim\end{array}$ & 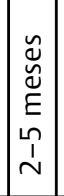 & 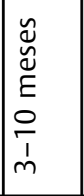 & 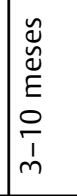 \\
\hline 苂 & - & - & $\sim$ & $\sim$ & $m$ & $m$ & $\nabla$ & in & in \\
\hline
\end{tabular}

Suporte Financeiro

Não houve suporte financeiro de fontes públicas, comerciais, ou sem fins lucrativos.

Conflito de interesses

Os autores declaram não haver conflito de interesses.

\section{Referências}

1 Goldfarb CA, Wall LB. Osteotomy for clinodactyly. J Hand Surg Am 2015;40(06):1220-1224

2 Piper SL, Goldfarb CA, Wall LB. Outcomes of opening wedge osteotomy to correct angular deformity in little finger clinodactyly. J Hand Surg Am 2015;40(05):908-13.e1

3 Medina JA, Lorea P, Elliot D, Foucher G. Correction of Clinodactyly by Early Physiolysis: 6-Year Results. J Hand Surg Am 2016;41(06): e123-e127

4 Ali M, Jackson T, Rayan GM. Closing wedge osteotomy of abnormal middle phalanx for clinodactyly. J Hand Surg Am 2009;34(05): 914-918

5 Cerqueiro-Mosquera J, Fleming AN. The bilobed flap: a new application in the reconstruction of congenital thumb deviation. J Hand Surg Br 2000;25(03):262-265

6 Strauss NL, Goldfarb CA. Surgical correction of clinodactyly: two straightforward techniques. Tech Hand Up Extrem Surg 2010;14 (01):54-57

7 Vickers D. Clinodactyly of the little finger: a simple operative technique for reversal of the growth abnormality. J Hand Surg $\mathrm{Br}$ 1987;12(03):335-342

8 Esser JF. Gestielte lokale nasenplastik mit zweizip̄igen lappen, deckung des sekundaÈ ren defektes vom ersten zipfel durch den zweiten. Deutsche Zeitschrift fur Chirurgie 1918;143:385-390

9 Evans DM, Gateley DR, Lewis JS. The use of a bilobed flap in the correction of radial club hand. J Hand Surg Br 1995;20(03): 333-337

10 Bednar MS, Bindra RR, Light TR. Epiphyseal bar resection and fat interposition for clinodactyly. J Hand Surg Am 2010;35(05):834-837

11 Anderson PJ, Hall CM, Smith PJ, et al. The hands in the Pfeiffer syndrome. J Hand Surg Br 1997;22:537-540

12 Wood VE, Rubinstein JH. Surgical treatment of the thumb in the Rubinstein-Taybi syndrome. J Hand Surg Br 1987;12(02):166-172

13 Varela Ares E, Yáñez J, Irisarri C. El tratamiento de la falange delta en el síndrome de Rubinstein-Taybi. A propósito de un caso. Acta Ortop Gallega 2012;8(02):65-67

14 Burke F, Flatt A. Clinodactyly. A review of a series of cases. Hand 1979;11(03):269-280

15 Carstam N, Theander G. Surgical treatment of clinodactyly caused by longitudinally bracketed diaphysis ("delta phalanx"). Scand J Plast Reconstr Surg 1975;9(03):199-202

16 Jain A, Rehman S, Smith G. Long-term results following osteotomy of the thumb delta phalanx in Rubinstein-Taybi Syndrome. J Hand Surg Eur Vol 2010;35(04):296-301

17 Jones GB. Delta Phalanx. J Bone Joint Surg Br 1964;46:226-228

18 Al-Qattan MM. Congenital sporadic clinodactyly of the index finger. Ann Plast Surg 2007;59(06):682-687

19 Young KA, Campbell AC. The bilobed flap in treatment of mucous cysts of the distal interphalangeal joint.J Hand Surg Br 1999;24(02):238-240

20 Jager T, Vogels J, Dautel G. The Zitelli design for bilobed flap applied on skin defects after digital mucous cyst excision. A review of 9 cases. Tech Hand Up Extrem Surg 2012;16(03):124-126

21 Zitelli JA. The bilobed flap for nasal reconstruction. Arch Dermatol 1989;125(07):957-959 